\section{Idiopathic Infantile Arterial Calcification: A Case Report}

\begin{abstract}
Keywords: Infant; Calcification; Artery
Abstract

Idiopathic Infantile Arterial Calcification (IIAC) is a rare autosomal recessive inherited disorder that influences the metabolism of pyrophosphate and leads to in-utero calcium deposition with fibrotic proliferation in the walls of the large and medium-sized arteries.

IIAC may present antenatally with polyhydramnios with or without arterial calcification, and may present postnatally if intractable systemic hypertension and/or heart failure from systemic hypertension and or ischemic coronary artery disease.

Diagnosis of IIAC is usually confirmed by the genetic study. It has a poor prognosis, with mortality been reported by the age of 4-6 months.

Increasing awareness about IIAC facilitates early diagnosis, early management, family counseling for prognosis and future conceptua plans, as early diagnosis helps in the decision of pregnancy termination.
\end{abstract}

\section{Introduction}

Idiopathic infantile arterial calcification (IIAC) is a rare inherited disorder in an autosomal recessive manner. IIAC has been described with an abnormal calcification of large and medium sized arteries with calcium deposition and fibrotic proliferation that take place in prenatal life. This disorder may affect renal arteries as well where babies may present with systemic hypertension $[1,2]$, pulmonary hypertension, ischemic heart failure and/or death in the first six months of life, children may present in different ages, but the prognosis is generally poor $[3,4]$.

\section{Case Presentation}

An un-booked 29 years old mother in the 32-week gestation, from a consanguineous marriage, was presented to our obstetric clinic for further evaluation of polyhydramnios. She was Gravida 4, Para 3 and all previous offsprings are healthy. Her obstetric history was unremarkable except for polyhydramnios with no known significant past medical or surgical history. Then she was admitted in labor for one day in the obstetric ward and Dexamethasone was administered as preparation of possible premature delivery, then cesarean section was performed because of fetal distress.

Postnatal examination revealed a male neonate small for gestational age with a body weight of $1 \mathrm{~kg}$. The baby was born flaccid with bradycardia that didn't improve by initial resuscitation and positive pressure ventilation with APGAR Score of 1 at 1 minute, 4 at 5 minutes and 7 at 10 minutes, so he was intubated soon after delivery and admitted to Neonatal Intensive Care Unit (NICU) where he received exogenous surfactant, umbilical catheters were placed, and total parenteral nutrition was started. Later, he developed hypertension which was controlled with intravenous hydrazine. Other physical exams were insignificant. Our workup revealed normal CBC, electrolyte, metabolic screening, lipid profile and negative blood culture growth.

\section{Journal of \\ Pediatrics \& Child Care}

\author{
Jalal Aldandan' ${ }^{1}$, Aminah Almoghannam¹, Ammar \\ Alkhars ${ }^{1}$ and Ghassan A. Shaath ${ }^{2 *}$ \\ ${ }^{\prime}$ Maternity and Children Hospital, Ministry of Health, Alahsa, Saudi \\ Arabia \\ ${ }^{2}$ King Abdulaziz Cardiac Center, Ministry of National Guard-Health \\ affairs, Saudi Arabia \\ *Address for Correspondence: \\ Ghassan A. Shaath, King Abdulaziz Cardiac Center, Ministry of \\ National Guard-Health affairs, Saudi Arabia, Tel: 00966535244333; \\ E-mail: shaathg@gmail.com \\ Submission: 15 February, 2017 \\ Accepted: 03 March, 2017 \\ Published: 08 March, 2017 \\ Copyright: ๑ 2017 Aldandan J, et al. This is an open access article \\ distributed under the Creative Commons Attribution License, which \\ permits unrestricted use, distribution, and reproduction in any medium, \\ provided the original work is properly cited.
}

Chest and abdominal X-ray consistent with Respiratory Distress Syndrome (RDS) with a normal cardiac silhouette, normal situs and normal gaseous distribution (Figure 1).

Abdominal and cranial ultrasound (US) showed calcification of lenticulostriate arteries with a normal splanchnic sonographic study, which also confirms the absence of renal arterial stenosis (Figure 2).

Electrocardiography showed normal Sinus rhythm and normal for age right QRS axis, with no evidence of increased Left Ventricle (LV) forces nor any evidence of ischemic myocardial changes.

Echocardiography shows situs solitus, levocardia with normal systemic and pulmonary venous connections and intracardiac anatomy as well, but with dense and hyperechoic Aortic arch, abdominal aorta, main pulmonary artery \& pulmonary artery branches with acoustic artifacts beyond the great vessels (Figures

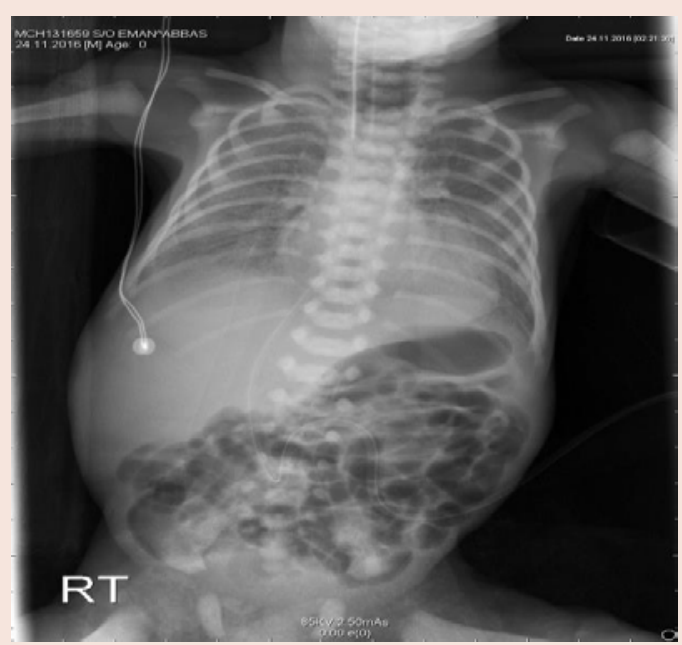

Figure 1: Reticulonodular opacity of both lungs with ground glass appearance (RDS). 
Citation: Aldandan J, Almoghannam A, Alkhars A, Shaath GA. Idiopathic Infantile Arterial Calcification: A Case Report. J Pediatr Child Care. 2017;3(1): 03.

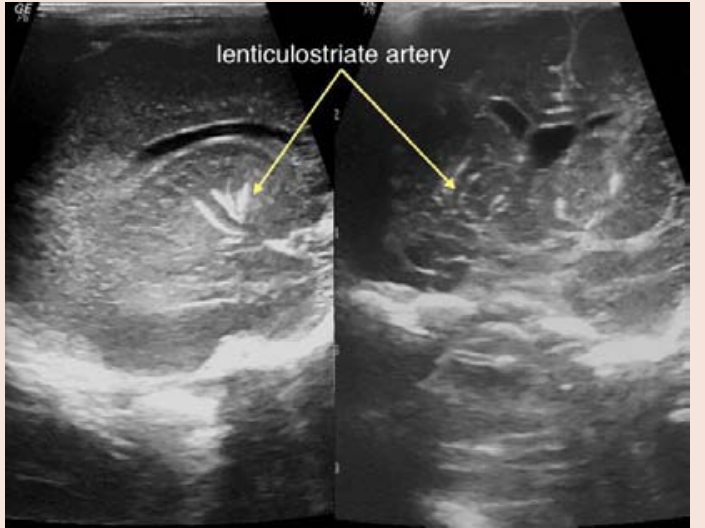

Figure 2: Calcification of lenticulostriate arteries.

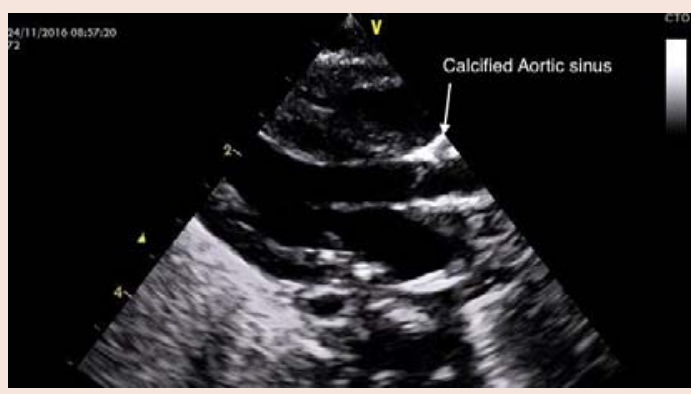

Figure 3: Calcification of aortic sinus.

3-6), indicating dense calcifications of the vascular walls.

There was no evidence of pericardial effusion. No Left Ventricle Outflow Obstruction (LVOTO) or Right Ventricle Outflow Obstruction (RVOTO), normal Left Ventricle (LV) posterior wall thickness in diastole (4 mm). Good biventricular systolic function.

Our finding of IIAC made us in the process of confirming the diagnosis genetically to counsel the family.

\section{Case Discussion}

IIAC has been described in the literature before as case reports but remains a rare disorder and was labeled with certain genetic abnormalities as an autosomal recessive disorder [1,2]. Which is unfortunately not available at the current time in our regional laboratory, but we are in the process to send it outside the country for genetic mapping including the parental genetic study to confirm the diagnosis and counsel the family after differentiating the de Novo mutation from inherited disorder, but that's expected to take months in our settings.

Nitschke and his colleagues were the first to describe the genetic abnormality with a mutation in the gene Ectonucleotide Pyrophosphatase Phosphodiesterase (ENPP1) which is the commonest genetic mutation described and located on the chromosome 6q2223 [5], genetic mapping is essential to confirm the diagnosis as this mutation disrupts the pyrophosphate metabolism. In the cases where genetic investigations do not help, the gold standard diagnosis is by histopathological findings with hydroxyapatite crystals deposition, which is difficult in our culture due to religious factors that prohibit autopsy procedure [6].

The associated polyhydramnios in the antenatal US is helpful to increase the suspicion of IIAC which was described in the previous literature but found to be a frequent finding, in addition to diagnosis that can be established antenatally as well [7]. Polyhydraminos was also our finding in the antenatal follow-up but unfortunately, we missed the evidence of calcified arteries early in the antenatal course probably as the calcium deposition did not take place. The clinical presentation may be otherwise related to heart failure such as pericardial effusion, congested lungs secondary to systemic hypertension with LV dysfunction and stiff great vessels or due to renal arteries involvement and/or due to ischemic changes in the coronary arteries calcifications.

Widespread arterial calcification is considered one of the characteristic finding of IIAC [2]. Coronary, aorta, carotid, mesenteric, and renal arteries usually involve in this process whereas cerebral vessels are usually spared [8]. Even though it's unusual, but there are other few reported cases with intracranial involvement [912]. Some cases presented with various manifestations include seizure, transient ischemic attacks, and strokes [2]. Unlike our case which had calcification of lenticulostriate arteries with no neurological manifestation.

It is necessary to identify index family to offer genetic counseling and molecular analysis to provide a prenatal diagnosis in subsequent conceptions [8].

Deferent medication had been described in the past such as estrogens, steroids, thyroid extract and bisphosphonates [13].

Several authors proposed using Bisphosphonate as a treatment as it reverses the abnormal arterial calcification $[2,8,14]$, it shows regression of calcifications after 4 months to 2 years therapy [14]. Peritoneal dialysis as a modality of treatment has been reported in a case of refractory hypertension [15]. Some cases reported as a spontaneous resolution of the calcifications, which makes the value of Bisphosphonate treatment questionable [8].

\section{Conclusion}

IIAC is a diagnosis of exclusion and should be suspected as a differential diagnosis for any baby with antenatal hydrops, polyhydramnios or signs of intractable systemic hypertension.

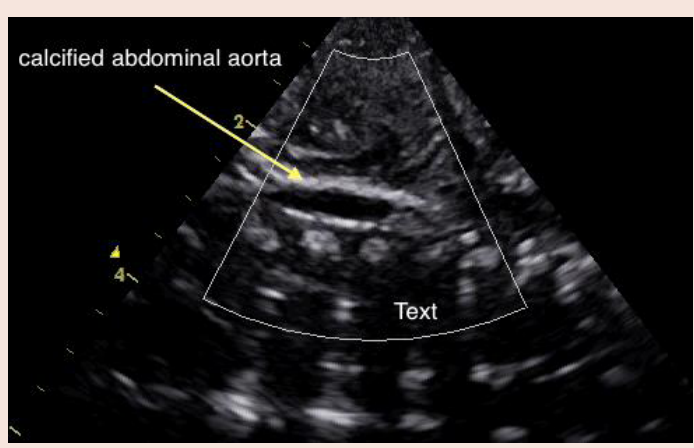

Figure 4: Calcification of abdominal aorta. 
Citation: Aldandan J, Almoghannam A, Alkhars A, Shaath GA. Idiopathic Infantile Arterial Calcification: A Case Report. J Pediatr Child Care. 2017;3(1): 03

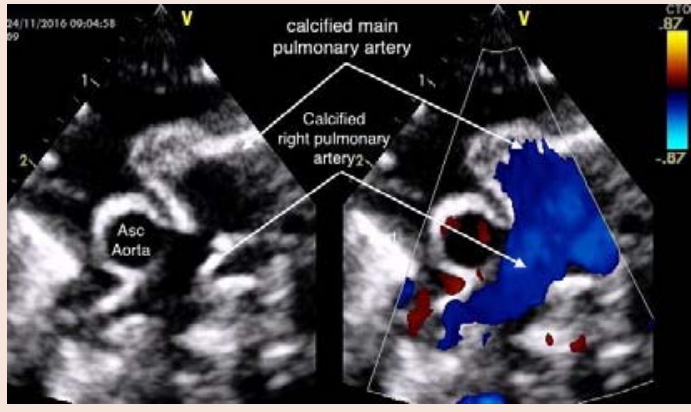

Figure 5: Calcification of main pulmonary artery and its branches.

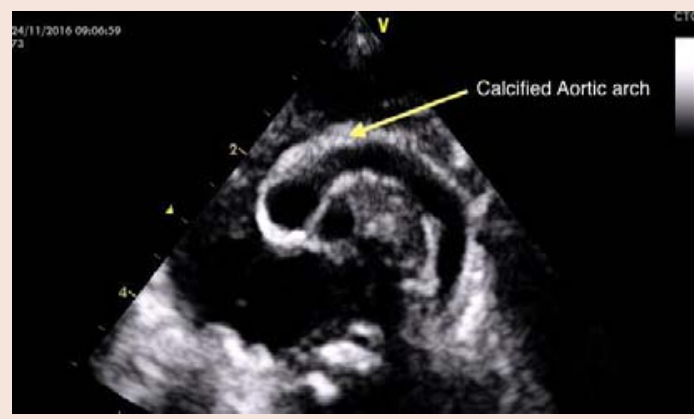

Figure 6: Calcification of aortic arch.

Confirming the diagnosis with genetic study and/or histopathological pyrophosphate crystals deposition would be a great help to counsel the families for future conceptions.

It is a necessary to identify index family in order to perform genetic analysis for prenatal diagnosis.

Further studies are needed to find out other treatment modalities.

\section{References}

1. Pejovic B, Peco-Antic A, Marinkovic-Eric J (2007) Blood pressure in noncritically ill preterm and full-term neonates. Pediatr Nephrol 22: 249-257.
2. Ferreira C, Ziegler S, Gahl W (1993) Generalized arterial calcification of infancy. In: Pagon RA, Adam MP, Ardinger HH, Wallace SE, Amemiya A, et al. (Eds). GeneReviews ${ }^{\circledR}$ [Internet]. Seattle (WA): University of Washington, Seattle.

3. Bolster F, Ali Z, Southall P, Fowler D (2015) Generalized arterial calcification of infancy--findings at post-mortem computed tomography and autopsy. Forensic Sci Int 254: e7-e12.

4. Amine M, Faten $\mathrm{H}$, Rim H, Nidhal HS, Njim L, et al. (2015) A rare cause of death in infancy: idiopathic infantile arterial calcification. Pathologica 107: 29-31.

5. Nitschke Y, Rutsch F (2012) Genetics in arterial calcification: lessons learned from rare diseases. Trends Cardiovasc Med 22: 145-149.

6. Shaireen H, Howlett A, Amin H, Yusuf K, Kamaluddeen M, et al. (2013) The mystery of persistent pulmonary hypertension: an idiopathic infantile arterial calcification. BMC Pediatr 13: 107

7. Nasrallah FK, Baho H, Sallout A, Qurashi M (2009) Prenatal diagnosis of idiopathic infantile arterial calcification with hydrops fetalis. Ultrasound Obstet Gynecol 34: 601-604.

8. Mastrolia SA, Weintraub AY, Baron J, Sciaky-Tamir Y, Koifman A et al (2015) Antenatal diagnosis of idiopathic arterial calcification: a systematic review with a report of two cases. Arch Gynecol Obstet 291: 977-986.

9. Sholler GF, Yu JS, Bale PM, Hawker RE, Celermajer JM, et al. (1984) Generalized arterial calcification of infancy: three case reports, including spontaneous regression with long-term survival. J Pediatr 105: 257-260.

10. Prior JT, Bergstrom VW (1948) Generalized arterial calcification in infants. Am J Dis Child 76: 91-101.

11. van der Sluis IM, Boot AM, Vernooij M, Meradji M, Kroon AA (2006) Idiopathic infantile arterial calcification: clinical presentation, therapy and long-term follow-up. Eur J Pediatr 165: 590-593.

12. Glatz AC, Pawel BR, Hsu DT, Weinberg P, Chrisant MRK (2006) Idiopathic infantile arterial calcification: two case reports, a review of the literature and a role for cardiac transplantation. Pediatr Transplant 10: 225-233.

13. Rosenbaum DM, Blumhagen JD (1986) Sonographic recognition of idiopathic arterial calcification of infancy. AJR Am J Roentgenol 146: 249-250.

14. Mahajan V, Sahni M, Dasgupta S, Amyn J, Jain SK (2016) Idiopathic calcification of infancy-case report and review of literature. J Pediatr Child Care 2: 1-5.

15. Stojanovic V, Doronjski A, Milak G, Barisic N (2013) Idiopathic arteria calcification of infancy-peritoneal dialysis for treatment of hypertension. Fetal Pediatr Pathol 32: 443-447. 